\title{
A MICROSTRUCTURE-BASED HYPERELASTIC MODEL FOR OPEN-CELL SOLIDS*
}

\author{
L. ANGELA MIHAI ${ }^{\dagger}$, HAYLEY WYATT ${ }^{\dagger}$, AND ALAIN GORIELY $\ddagger$
}

\begin{abstract}
Mesoscopic continuum hyperelastic models for open-cell solids subject to large elastic deformations are derived from the architecture of the cellular body and the microscopic responses of the cell walls. These models are valid for general structures, with randomly oriented cell walls, made from an arbitrary isotropic nonlinear hyperelastic material, and subject to finite triaxial stretches. Their analyses provide global descriptors of the cellular structure, such as nonlinear stretch and shear moduli, and Poisson's ratio. Comparisons with numerical simulations show that the mesoscopic models capture well the mechanical responses under large strain deformations of three-dimensional periodic structures and of two-dimensional honeycombs made from a neo-Hookean material.
\end{abstract}

Key words. cellular solids, microstructural behavior, constitutive modelling, open cells, hyperelastic material, large strain deformation

AMS subject classifications. 74A05, 74A20, 74A40, 74B20

DOI. $10.1137 / 16 \mathrm{M} 1098899$

1. Introduction. Cellular solids, or foams, are two- or three-dimensional bodies divided into cells, which may be open or closed, and can be filled with a fluid or solid core. In open-celled solids, the cell walls consist of the cell edges which form an interconnected network, while in closed-cell foams, the cell walls contain both the cell edges and the cell faces forming disconnected cell compartments, though some foams may contain both open and closed cells [12, 16, 17, 42]. Since a foam (spuma in Latin; $\alpha \varphi \rho o ́ \varsigma$ in Greek) is not a continuum, but its mechanical properties depend on the intrinsic material characteristics of the cell walls and on the size and shape of the cells, a continuum theory cannot account for all microstructural effects. Nevertheless, even though it is at a cellular level that the structural basis of foams is best addressed, both theoretically and computationally, due to the inherent complexity and diversity of cellular bodies, the mathematical modelling of every individual cell in a structure with a large number of cells is generally unfeasible, and a combined study spanning the micro-, meso-, and macroscopic scales is required.

Since many cellular solids return to their initial shape after loads are removed, at low stresses or strains, the assumption that the cell walls are linearly elastic with a geometrically nonlinear behavior is valid, and is successfully used in structural mechanics and other engineering applications, e.g., wood, metal, or elastomeric foams, or structural ceramics. In this case, if the deformation is primarily by cell-wall bending, the elastic responses can be estimated from the linear-elastic deflection of a beam $[6,16,17]$. In particular, for honeycomb structures with uniform hexagonal cells loaded uniaxially in plane, assuming that the cell walls, with thickness $t$ and length $L$, initially deform linearly elastically by bending, without stretching, the Young's

\footnotetext{
*Received by the editors October 14, 2016; accepted for publication (in revised form) June 6, 2017; published electronically August 22, 2017.

http://www.siam.org/journals/siap/77-4/M109889.html

Funding: The first and second author's work was supported by the Engineering and Physical Sciences Research Council of Great Britain under research grant EP/M011992/1.

${ }^{\dagger}$ School of Mathematics, Cardiff University, Senghennydd Road, Cardiff, CF24 4AG, UK (MihaiLA@cardiff.ac.uk, WyattHL@cardiff.ac.uk).

${ }^{\ddagger}$ Mathematical Institute, University of Oxford, Woodstock Road, Oxford, OX2 6GG, UK (goriely@maths.ox.ac.uk).
} 
modulus is proportional to $(t / L)^{3}$, while the relative density $\rho^{*} / \rho_{s}$, where $\rho^{*}$ is the density of the cellular structure and $\rho_{s}$ is the density of the solid cell wall material, is proportional to $t / L[17$, pp. 34-35]. Similarly, for three-dimensional cellular solids with uniform open cubical cells in staggered distribution and subject to uniaxial loading, such that the cell edges deform by linear-elastic bending, the Young's modulus is proportional to $(t / L)^{4}$, or equivalently, to $\left(\rho^{*} / \rho_{s}\right)^{2}$ [17, pp. 44-48]. However, for stretch-dominated prismatic architectures, which are structurally more efficient, due to a higher stiffness-to-weight ratio than the bending-dominated ones $[9,10]$, the Young's modulus computed from the stretch deformation of the pin-jointed cell walls is proportional to $\rho^{*} / \rho_{s}$, or equivalently, to $(t / L)^{2}$, while the buckling stress is proportional to $\left(\rho^{*} / \rho_{s}\right)^{2}[17$, pp. 57-58].

Many modern applications (e.g., synthetic rubbers) and biological structures (e.g., soft tissues and organs) also involve large strains, whereby the deformation is inherently nonlinear and the corresponding stresses depend on both the position and the underlying material properties [28]. At the individual cell level, for many natural and engineered cellular structures, physical evidence suggests that several main factors determine the magnitude of the enhancement of stress level in the cellular body, including the cell geometry, the cell wall thickness, and the number of cells $[1,11,13,37]$. In [27], for honeycomb structures of nonlinear hyperelastic material with standard cellgeometry, it was shown that if a structure contains walls which are inclined relative to the applied uniaxial tensile load, then the apparent Poisson's ratio depends on the deformation and decreases as the magnitude of the tensile load increases. In [25, 26], a nonlinear elastic modulus was identified and, under increasing strain, was shown to increase as the thickness of the cell wall increases or when the wall is multilayer, as well as when the number of cells increases while the volume of cell wall material and the ratio between the thickness and the length of the wall remain fixed.

Understanding the properties and local behavior of the structural components is essential when continuum models are designed to predict responses in cellular solids at a macroscopic level. The first constitutive model for the mechanical response of a cellular solid deduced from the microstructural cell model is due to Gent and Thomas (1959) [14]. For this model, general isotropic linearly elastic open-cell foams subject to small stretch deformations were assumed, and effective Young's elastic modulus and the Poisson's ratio were derived directly from the constitutive equations $[2,4]$. This model was later extended to closed-cell foams containing an ideal gas [15]. For the study of the nonlinearly elastic response of a compressible isotropic cellular material under finite strain, a phenomenological continuum model is due to Blatz and Ko (1962) [7]. This model reduces to the Gent-Thomas model in the small strain regime, and for both the Gent-Thomas and the Blatz-Ko models, the effective Poisson's ratio has a fixed value of $1 / 4$ [5]. The derivation from continuum mechanics of a general class of constitutive equations of the Blatz-Ko type may be found in the review article [3]. In [40], it was noted that Hill's energy functional of hyperelasticity [19] can be used to describe the simple special case of foams where the principal stresses are uncoupled, i.e., depend only upon the stretch ratio in the corresponding principal direction. These approaches are based on the strain-energy function for incompressible media proposed by Ogden (1972) [35] which is extended to the compressible case. Hyperelastic models are described by a strain-energy density function [36, 41], and are generally amenable to computer simulations based on the finite element method $[23,34]$.

In this study, assuming that the cell walls are circular cylinders and are subject to finite triaxial stretches, and the joints between adjacent walls are spheres with the radius much smaller than the length of the wall, and do not deform signifi- 
cantly, continuum isotropic hyperelastic models for open-cell solids at the mesoscopic scale comprising a finite number of cells are developed that account for the geometry and the material constitutive law of the structural elements (section 2). Unlike phenomenological models that provide effective elastic parameters based on macroscopic observations and measurements, the constitutive parameters for these models are derived explicitly from the strain-energy function of the underlying solid material and the large strains in the cell walls. Specifically, nonlinear stretch and shear moduli are computed under simple axial and shear deformations, respectively, and a Poisson's ratio is obtained under simple compression or tension (section 3). For illustrative purposes, the case of neo-Hookean cell walls is treated in details (section 4). Furthermore, when compression of the continuum hyperelastic material takes place parallel to the direction of some of the cell walls, the material critical stretches and stresses for the onset of nonlinear Euler buckling of the cell walls are derived from the critical stretches for the cell walls under axial load provided by Goriely, Vandiver, and Destrade (2008) [18]. To assess their mechanical performance, the continuum models are compared numerically to computer simulations of three-dimensional periodic structures and of two-dimensional honeycombs of neo-Hookean material in large tension (section 5). Theoretically, the continuum models developed here are valid also for more general structures, with randomly oriented cell walls made from an arbitrary isotropic nonlinear hyperelastic material.

Under uniform stresses, many isotropic materials deform uniformly [29, 30, 33], whereas in cellular structures, the deformation generally concentrates in regions that are more easily deformable. The hyperelastic models developed here capture structural behaviors at a mesoscopic level, where the number of cells is finite and the size of the structure is comparable to the cell-size, and can be useful as an initial approximation in multilevel approaches, whereby a cellular structure is represented first as an elastic material deforming uniformly, and after the loading is increased, the areas where the stress field reaches critical values are remodelled as individual cells to predict critical local effects [31].

2. Hyperelastic models for structures with open cells. In this section, we present the approach for constructing strain-energy functions for homogeneous isotropic hyperelastic models of cellular structures with open cells and identical but randomly oriented cell walls, provided that the cell walls are circular cylinders and are subject to finite triaxial stretches, without bending or buckling, and the joints between adjacent walls are spheres with the radius much smaller than the length of the walls and do not deform significantly. These geometrical conditions are similar to those for the Gent-Thomas model [14], where the cell walls were treated as linearly elastic. However, in our models, the cell walls are made from a general isotropic hyperelastic material and are capable of finite strain deformations. The triaxial stretch of the cell walls may occur, for example, when the collective deformation of a finite number of cells can be reasonably approximated as homogeneous, in which case the elastic responses of those cells can be captured by a continuum model.

To derive the continuum model, we first relate the principal stretches at the mesoscopic scale to those of the individual cell walls, then formulate the strain-energy density at the mesoscopic level as a function of the principal stretches of the cell walls by averaging over a unit volume of material. The unit volume is taken as either a sphere or a finite number of spheres, with each sphere centered at the intersection of adjacent walls, but the same results are obtained if the unit volume is cubical in shape with the cuboid walls aligning with the cube edges and the cubical joints 
placed at the cube corners. To show how the stiffness of the cellular body at the mesoscopic scale changes with the ratio between the volume of solid material and that of the elastic structure, this model is specialized to the case where the volume fraction of solid material contained in a unit volume of cellular structure depends only on the ratio between the thickness and the length of the cell walls. Moreover, under finite strain deformations, if both the volume fraction of solid material and that of the elastic structure and the ratio between the thickness and the length of the cell walls remain fixed, then structures with a larger number of smaller cells may become stiffer compared to those with fewer and larger cells. To capture this behavior, the strain-energy function is augmented with a suitable weight, such that the resulting elastic modulus increases if the number of cells increases while the volume fraction and the ratio between the thickness and the length of the walls are fixed.

2.1. Cell walls under finite triaxial stretch. For a cellular structure with open cells and randomly oriented cell walls, we denote by $L$ the initial length of a cell wall, and by $t$ the width of the joint between adjacent walls, such that $0<k=t / L<1$. We further denote by $l$ the length of the finitely deformed cell wall, and assume that the deformation of the joints is less significant than that of the cell walls, and hence the deformation of the joints can be neglected. We also assume that, in the undeformed state, the wall thickness is approximately equal to the width of the joint. Then $\bar{L}=L+t=(1+k) L$ and $\bar{l}=l+t=l+k L$ are the lengths of a cell element comprising a cell wall and a joint before and after the deformation, respectively, and denoting by $\left\{\lambda_{i}\right\}_{i=1,2,3}$ and $\left\{\bar{\lambda}_{i}\right\}_{i=1,2,3}$ are the principal stretches for the cell wall and the cell element, respectively, we obtain

$$
\lambda_{i}=(1+k) \bar{\lambda}_{i}-k, \quad i=1,2,3 .
$$

Let $\left(\mathbf{e}_{1}, \mathbf{e}_{2}, \mathbf{e}_{3}\right)$ be the usual orthonormal vectors for the Cartesian coordinates in the principal directions of the material deformation at the mesoscopic scale, and let $\left(\mathbf{n}_{1}, \mathbf{n}_{2}, \mathbf{n}_{3}\right)$ denote the orthonormal vectors in the principal direction of the deforming cell wall, as shown in Figure 1, such that

$$
\begin{aligned}
& \mathbf{n}_{1}=-\mathbf{e}_{1} \cos \theta \cos \phi-\mathbf{e}_{2} \cos \theta \sin \phi+\mathbf{e}_{3} \sin \theta, \\
& \mathbf{n}_{2}=\mathbf{e}_{1} \sin \phi-\mathbf{e}_{2} \cos \phi \\
& \mathbf{n}_{3}=\mathbf{e}_{1} \sin \theta \cos \phi+\mathbf{e}_{2} \sin \theta \sin \phi+\mathbf{e}_{3} \cos \theta .
\end{aligned}
$$

Denoting by $\left\{\alpha_{i}\right\}_{i=1,2,3}$ the principal stretches for the cellular solid at the mesoscopic scale, and setting $\mathbf{F}_{m}=\operatorname{diag}\left(\alpha_{1}, \alpha_{2}, \alpha_{3}\right)$, we can write

$$
\begin{aligned}
& \bar{\lambda}_{1}=\mathbf{n}_{1} \cdot \mathbf{F}_{m} \mathbf{n}_{1}=\alpha_{1} \cos ^{2} \theta \cos ^{2} \phi+\alpha_{2} \cos ^{2} \theta \sin ^{2} \phi+\alpha_{3} \sin ^{2} \theta, \\
& \bar{\lambda}_{2}=\mathbf{n}_{2} \cdot \mathbf{F}_{m} \mathbf{n}_{2}=\alpha_{1} \sin ^{2} \phi+\alpha_{2} \cos ^{2} \phi \\
& \bar{\lambda}_{3}=\mathbf{n}_{3} \cdot \mathbf{F}_{m} \mathbf{n}_{3}=\alpha_{1} \sin ^{2} \theta \cos ^{2} \phi+\alpha_{2} \sin ^{2} \theta \sin ^{2} \phi+\alpha_{3} \cos ^{2} \theta .
\end{aligned}
$$

We assume that the cell walls are made from a homogeneous isotropic hyperelastic material described by a strain-energy density function $\mathcal{W}\left(\lambda_{1}, \lambda_{2}, \lambda_{3}\right)$. For the deforming cell wall, which is subject to a triaxial stretch, the non-zero components of the first Piola-Kirchhoff stress tensor are the diagonal ones, i.e.,

$$
P_{i}=\frac{\partial \mathcal{W}}{\partial \lambda_{i}}, \quad i=1,2,3
$$




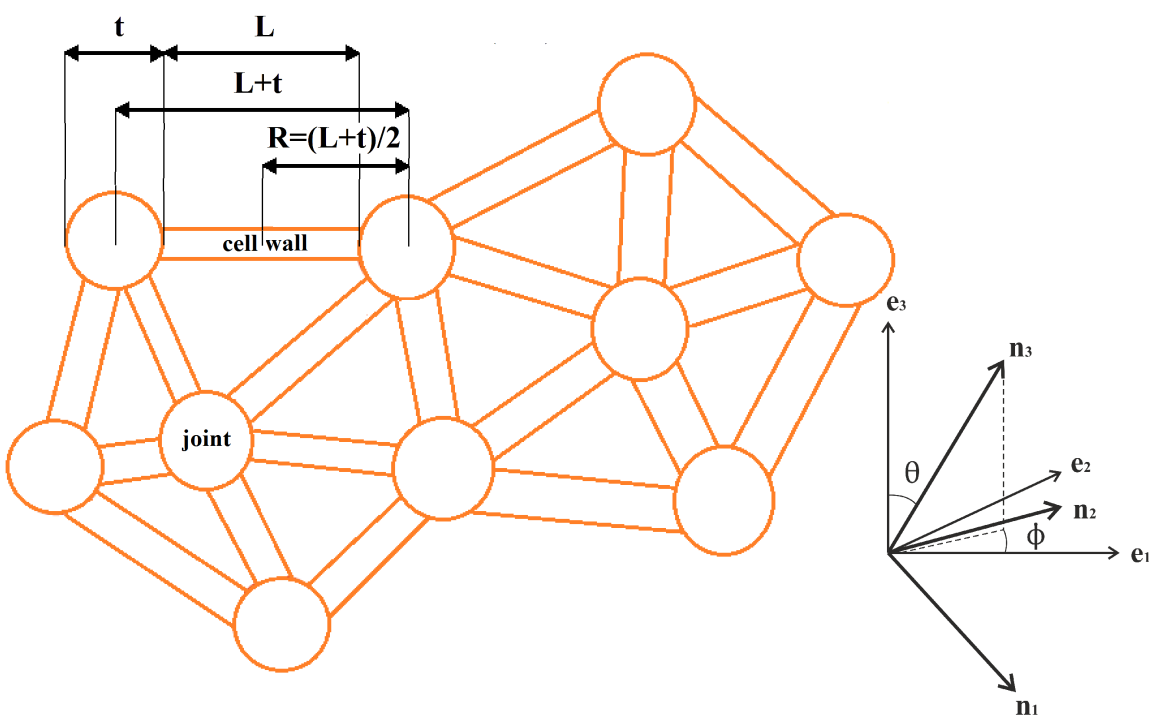

FIG. 1. Schematic of cell walls and joints in a cellular structure, with the orthonormal vectors $\left(\boldsymbol{e}_{1}, \boldsymbol{e}_{2}, \boldsymbol{e}_{3}\right)$ for the structure at the mesoscopic scale and $\left(\boldsymbol{n}_{1}, \boldsymbol{n}_{2}, \boldsymbol{n}_{3}\right)$ for a cell wall.

and the associated components of the Cauchy stress tensor are

$$
\sigma_{i}=J^{-1} \lambda_{i} P_{i}=J^{-1} \lambda_{i} \frac{\partial \mathcal{W}}{\partial \lambda_{i}}=J^{-1} \frac{\partial \mathcal{W}}{\partial\left(\ln \lambda_{i}\right)}, \quad i=1,2,3,
$$

where $J=\lambda_{1} \lambda_{2} \lambda_{3}$. The condition on the strain-energy function $\mathcal{W}$ for $\lambda_{1}=\lambda_{2}=$ $\lambda_{3}=1$ to be a minimum is

$$
\frac{\partial \mathcal{W}^{(m)}}{\partial\left(\ln \lambda_{i}\right)}=0, \quad i=1,2,3
$$

We define the following principal invariants of the stretch tensor for the cell element [39]:

$$
\bar{i}_{1}=\bar{\lambda}_{1}+\bar{\lambda}_{2}+\bar{\lambda}_{3}, \quad \bar{i}_{2}=\bar{\lambda}_{1} \bar{\lambda}_{2}+\bar{\lambda}_{2} \bar{\lambda}_{3}+\bar{\lambda}_{3} \bar{\lambda}_{1}, \quad \bar{i}_{3}=\bar{\lambda}_{1} \bar{\lambda}_{2} \bar{\lambda}_{3} .
$$

Then, using (2.1), the strain-energy function describing the cell wall material can be written equivalently as $\mathcal{W}\left(\lambda_{1}, \lambda_{2}, \lambda_{3}\right)=\overline{\mathcal{W}}\left(\bar{i}_{1}, \bar{i}_{2}, \bar{i}_{3}\right)$, regardless of $k$, since the deformation of the joints is negligible.

We denote the principal invariants of the stretch tensor for the cellular solid at the mesoscopic level by

$$
i_{1}=\alpha_{1}+\alpha_{2}+\alpha_{3}, \quad i_{2}=\alpha_{1} \alpha_{2}+\alpha_{2} \alpha_{3}+\alpha_{3} \alpha_{1}, \quad i_{3}=\alpha_{1} \alpha_{2} \alpha_{3} .
$$

By the change of frame (2.3), the principal invariants of the stretch tensor for the cell element are the same as the principal invariants of the stretch tensor for the cellular solid, i.e.,

$$
\bar{i}_{1}=i_{1}, \quad \bar{i}_{2}=i_{2}, \quad \bar{i}_{3}=i_{3},
$$


as expected. Therefore, $\overline{\mathcal{W}}\left(\bar{i}_{1}, \bar{i}_{2}, \bar{i}_{3}\right)=\overline{\mathcal{W}}\left(i_{1}, i_{2}, i_{3}\right)$, with no explicit dependence on the angles $(\theta, \phi)$.

Then the strain energy per unit volume of the cellular solid at the mesoscopic level (designated by a subscript or superscript $m$ ) can be derived by taking the mean value of the cell wall energy $\mathcal{W}$ over the unit sphere, i.e.,

$$
\begin{aligned}
\mathcal{W}^{(m)} & =N V \frac{2}{\pi} \int_{0}^{\pi / 2} \int_{0}^{\pi / 2} \overline{\mathcal{W}}\left(i_{1}, i_{2}, i_{3}\right) \sin \theta \mathrm{d} \theta \mathrm{d} \phi \\
& =N V \overline{\mathcal{W}}\left(i_{1}, i_{2}, i_{3}\right),
\end{aligned}
$$

where $N$ is the number of full cell walls in a unit volume of cellular material and $V$ is the volume of a cell wall. The strain-energy function (2.11) describes the behavior of the deforming cellular solid at the mesoscopic scale, provided that the cell walls are subject to finite triaxial stretch, without bending or buckling.

For the deforming cellular solid, the principal components of the first PiolaKirchhoff stress tensor

$$
P_{i}^{(m)}=\frac{\partial \mathcal{W}^{(m)}}{\partial \alpha_{i}}, \quad i=1,2,3
$$

and the principal components of the Cauchy stress tensor are

$$
\sigma_{i}^{(m)}=J_{m}^{-1} \alpha_{i} P_{i}^{(m)}=J_{m}^{-1} \alpha_{i} \frac{\partial \mathcal{W}^{(m)}}{\partial \alpha_{i}}=J_{m}^{-1} \frac{\partial \mathcal{W}^{(m)}}{\partial\left(\ln \alpha_{i}\right)}, \quad i=1,2,3,
$$

where $J_{m}=\alpha_{1} \alpha_{2} \alpha_{3}$. The minimum strain energy given by the relations

$$
\frac{\partial \mathcal{W}^{(m)}}{\partial\left(\ln \alpha_{i}\right)}=0, \quad i=1,2,3,
$$

corresponds to the unstressed state, and by (2.13) and (2.6) it is attained if $\alpha_{1}=$ $\alpha_{2}=\alpha_{3}=1$, i.e., when the cellular body is undeformed.

2.2. The effect of volume fraction. To investigate the effect of the volume ratio between the elastic solid and the cellular material, we assume that $n$ cell walls, each of undeformed length $L$ and volume $V$, are meeting at a common joint of surface area

$$
n A=4 \pi(t / 2)^{2}=\pi k^{2} L^{2},
$$

where

$$
k=\frac{t}{L}=\sqrt{\frac{n A}{\pi L^{2}}} .
$$

Taking a representative volume of the cellular material at the mesoscopic scale in the undeformed state as a sphere of radius $R=(L+t) / 2=(1+k) L / 2$ centered at a joint, as indicated in Figure 1, the volume of this sphere is equal to

$$
\bar{V}_{m}=\frac{4 \pi R^{3}}{3}=\frac{\pi L^{3}(1+k)^{3}}{6},
$$

while the volume of solid material contained in this sphere is

$$
\bar{V}=\frac{n A L}{2}+\frac{4 \pi(t / 2)^{3}}{3}=\frac{\pi k^{2} L^{3}}{2}+\frac{\pi k^{3} L^{3}}{6}=\frac{\pi k^{2} L^{3}(3+k)}{6} .
$$

Copyright $@$ by SIAM. Unauthorized reproduction of this article is prohibited. 
By (2.14) and (2.15), the volume ratio is equal to

$$
\rho_{m}=\bar{V} / \bar{V}_{m}=1-\frac{1+3 k}{(1+k)^{3}},
$$

and increases as the parameter $k \in(0,1)$ increases.

Without loss of generality, setting the unit sphere of cellular material at the mesoscopic scale as $\bar{V}_{m}=1$, by (2.14), it follows that

$$
\frac{\pi L^{3}}{2}=\frac{3}{(1+k)^{3}},
$$

which implies that the volume fraction of cell wall material in this sphere is equal to

$$
N V=\frac{n A L}{2}=\frac{\pi k^{2} L^{3}}{2}=\frac{3 k^{2}}{(1+k)^{3}} .
$$

Alternatively, setting the representative volume of the cellular body at the mesoscopic scale, in the undeformed state, as $n_{m}$ spheres of radius $R=(L+t) / 2=(1+k) L / 2$, such that each sphere is centered at a joint, this volume is equal to $n_{m} \bar{V}_{m}$, where $\bar{V}_{m}$ is given by (2.14), and contains $n_{m} \bar{V}$ volume of solid material, with $\bar{V}$ defined by (2.15). Therefore, the corresponding volume ratio is the same as that defined by (2.16), since

$$
\rho_{m}=\left(n_{m} \bar{V}\right) /\left(n_{m} \bar{V}_{m}\right)=\bar{V} / \bar{V}_{m}=1-\frac{1+3 k}{(1+k)^{3}} .
$$

Taking the unit sphere of cellular material at the mesoscopic scale as $n_{m} \bar{V}_{m}=1$ implies

$$
\frac{\pi L^{3}}{2}=\frac{3}{n_{m}(1+k)^{3}},
$$

hence the associated volume fraction of cell wall material is equal to that given by (2.17), since

$$
N V=\frac{n_{m} n A L}{2}=\frac{n_{m} \pi k^{2} L^{3}}{2}=\frac{3 k^{2}}{(1+k)^{3}} .
$$

We conclude that the volume fraction of cell wall material contained in a unit volume of cellular structure depends only on the parameter $k$ representing the ratio between the thickness and the length of a cell wall. The same results are obtained if the unit volume is cubical in shape with the cuboid walls aligning with the cube edges and the cubical joints placed at the cube corners. This case is discussed in detail in section 5, where numerical examples are presented.

In the subsequent analysis, we maintain the notation $N V$ for the volume fraction of cell wall material in a representative unit volume in general, and use the particular case (2.17) as an example.

3. Nonlinear elastic moduli. In this section, for the cellular solid at the mesoscopic scale, the elastic moduli are derived from the elastic moduli of the cell wall material.

3.1. Shear modulus. To compute the nonlinear shear modulus for the cell wall and the cellular solid, respectively, we consider each of them under the simple shear deformation

$$
x_{1}=X_{1}, \quad x_{2}=X_{2}, \quad x_{3}=\gamma X_{1}+X_{3},
$$


where $\left(X_{1}, X_{2}, X_{3}\right)$ denote the Lagrangian (reference) and $\left(x_{1}, x_{2}, x_{3}\right)$ are the Eulerian (current) coordinates, respectively, and the constant $\gamma>0$ is the amount of shear. Then, the corresponding principal stretches satisfy

$$
\lambda_{1}^{2}=1+\frac{\gamma^{2}-\gamma \sqrt{\gamma^{2}+4}}{2}=\alpha^{-2}, \quad \lambda_{2}^{2}=1, \quad \lambda_{3}^{2}=1+\frac{\gamma^{2}+\gamma \sqrt{\gamma^{2}+4}}{2}=\alpha^{2},
$$

and similarly,

$$
\alpha_{1}^{2}=\alpha^{-2}, \quad \alpha_{2}^{2}=1, \quad \alpha_{3}^{2}=\alpha^{2}
$$

Noting that

$$
\alpha=\frac{\gamma+\sqrt{\gamma^{2}+4}}{2} \quad \text { and } \quad \alpha^{-1}=-\frac{\gamma-\sqrt{\gamma^{2}+4}}{2}
$$

we can compute the respective principal Cauchy stress components using (2.5) and (2.13). Then, under the deformation (3.1), the nonlinear shear modulus [41, p. 175] for the cell wall and the cellular solid at the mesoscopic scale are defined, respectively, as follows:

$$
\begin{aligned}
& \mu=\frac{\sigma_{3}-\sigma_{1}}{\alpha^{2}-\alpha^{-2}}, \\
& \mu^{(m)}=\frac{\sigma_{3}^{(m)}-\sigma_{1}^{(m)}}{\alpha^{2}-\alpha^{-2}} .
\end{aligned}
$$

In the limit of small shear strain, the corresponding shear moduli for the cell walls and the cellular solid take the form

$$
\begin{aligned}
& \mu_{0}=\lim _{\gamma \rightarrow 0} \mu, \\
& \mu_{0}^{(m)}=\lim _{\gamma \rightarrow 0} \mu^{(m)},
\end{aligned}
$$

and a direct computation of these moduli gives

$$
\begin{aligned}
& \mu_{0}=2\left(W_{010}+W_{100}\right), \\
& \mu_{0}^{(m)}=(1+k)^{2} N V \mu_{0},
\end{aligned}
$$

where

$$
W_{i j k}=\left.\left(\frac{\partial^{i} \partial^{j} \partial^{k}}{\partial I_{1}^{j} \partial I_{2}^{j} \partial I_{3}^{k}} \mathcal{W}\left(I_{1}, I_{2}, I_{3}\right)\right)\right|_{\left(I_{1}=3, I_{2}=3, I_{3}=1\right)},
$$

with

$$
I_{1}=\lambda_{1}^{2}+\lambda_{2}^{2}+\lambda_{3}^{2}, \quad I_{2}=\lambda_{1}^{2} \lambda_{2}^{2}+\lambda_{2}^{2} \lambda_{3}^{2}+\lambda_{3}^{2} \lambda_{1}^{2}, \quad I_{3}=\lambda_{1}^{2} \lambda_{2}^{2} \lambda_{3}^{2},
$$

and the positive indices $i, j, k$ assumed to be such that $i+j+k$ is between 0 and 2 .

When $N V$ is given by (2.17), the shear modulus for the cellular solid takes the form

$$
\mu_{0}^{(m)}=\frac{3 k^{2}}{1+k} \mu_{0}
$$

Copyright (C) by SIAM. Unauthorized reproduction of this article is prohibited. 
3.2. Stretch modulus. Another important descriptor of material response of an elastic body is the stretch modulus. To compute the stretch modulus for the wall, we consider a uniaxial extension with stretches $\left(\lambda_{1}(\lambda), \lambda_{1}(\lambda), \lambda\right)$ with the condition $\sigma_{1}=\sigma_{2}=0$ and $\sigma_{3}=N(\lambda)$. Similarly, for the cellular solid, we have stretches $\left(\alpha_{1}(\alpha), \alpha_{1}(\alpha), \alpha\right)$ with the condition $\sigma_{1}^{(m)}=\sigma_{2}^{(m)}=0$ and $\sigma_{3}^{(m)}=N^{(m)}(\alpha)$. The nonlinear stretch modulus is defined as the slope of the curve representing the axial stress versus the associated logarithmic strain, i.e.,

$$
\begin{aligned}
& E=\frac{\partial N(\lambda)}{\partial(\ln \lambda)}, \\
& E^{(m)}=\frac{\partial N^{(m)}(\alpha)}{\partial(\ln \alpha)} .
\end{aligned}
$$

In the linear elastic limit, each modulus (3.11)-(3.12) reduces to the corresponding Young's modulus for the individual wall and the cellular solid, respectively,

$$
\begin{aligned}
& E_{0}=\lim _{\lambda \rightarrow 1} E, \\
& E_{0}^{(m)}=\lim _{\alpha \rightarrow 1} E^{(m)} .
\end{aligned}
$$

We can find an explicit expression for these moduli by computing $\lambda_{1}(\alpha)$ in the small strain limit to obtain

$$
\begin{aligned}
& \lambda_{1}=1-(\lambda-1) \frac{W_{002}-W_{010}+4 W_{011}+4 W_{020}-W_{100}+2 W_{101}+4 W_{110}+W_{200}}{2 W_{002}-W_{010}+8 W_{011}+8 W_{020}-W_{100}+4 W_{101}+8 W_{110}+2 W_{200}} \\
& \quad+\mathcal{O}\left((\lambda-1)^{2}\right),
\end{aligned}
$$

where $W_{i j k}$, with $i, j, k$ such that $0 \leq i+j+k \leq 2$, is given by (3.8).

Then, the Young's modulus for the wall is

$$
E_{0}=2 \mu_{0} \frac{3 W_{002}-2 W_{010}+12 W_{011}+12 W_{020}-2 W_{100}+6 W_{101}+12 W_{110}+3 W_{200}}{2 W_{002}-W_{010}+8 W_{011}+8 W_{020}-W_{100}+4 W_{101}+8 W_{110}+2 W_{200}} .
$$

Performing a similar computation for the cellular solid, the Young's modulus of the cellular solid can be expressed in terms of the Young's modulus of the elastic walls by

$$
E_{0}^{(m)}=(1+k)^{2} N V E_{0} .
$$

In particular, if $N V$ is given by (2.17), then

$$
E_{0}^{(m)}=\frac{3 k^{2}}{1+k} E_{0}
$$

This linear elastic modulus is thus proportional to $k^{2}$, in agreement with the Young's modulus for stretch-dominated prismatic structures described by [17, pp. 57-58] when $k \ll 1$.

3.3. Poisson's ratio. For an elastic material which is finitely extended or compressed in the third direction and deforms freely in the orthogonal directions, the nonlinear Poisson's ratio can be computed as the negative quotient of the logarithmic strain in an orthogonal direction to that of the logarithmic strain in the third 
direction [5]. For the cell wall and the cellular body, respectively, the Poisson's ratio is

$$
\begin{aligned}
& \nu=-\frac{\ln \lambda_{1}}{\ln \lambda_{3}}, \\
& \nu^{(m)}=-\frac{\ln \alpha_{1}}{\ln \alpha_{3}} .
\end{aligned}
$$

Assuming that uniaxial loading causes a simple tension or compression in the direction of the tensile force, we can, in principle, express $\lambda_{1}=\lambda_{2}$ and $\alpha_{1}=\alpha_{2}$ as functions of $\lambda_{3}=\lambda>0$ and $\alpha_{3}=\alpha>0$, respectively. Therefore, in the limit of small strains, we recover the classic definition of the Poisson's ratio as

$$
\begin{aligned}
& \nu_{0}=-\lim _{\lambda \rightarrow 1} \frac{\ln \lambda_{1}(\lambda)}{\ln \lambda}, \\
& \nu_{0}^{(m)}=-\lim _{\alpha \rightarrow 1} \frac{\ln \alpha_{1}(\alpha)}{\ln \alpha} .
\end{aligned}
$$

To compute the Poisson's ratio for the wall, we use the condition $\sigma_{1}=\sigma_{2}=0$ to obtain (3.15), so that (3.21) and the expressions for the Young's and shear moduli give

$$
\nu_{0}=\nu_{0}^{(m)}=\frac{E_{0}-2 \mu_{0}}{2 \mu_{0}},
$$

as expected from the classic linear theory of elasticity [22]. Thus, under small strains, the Poisson's ratio of the cellular body is independent of the architecture and directly inherited from the elastic response of the cell wall.

3.4. The cell-size effect. So far, the material parameters for the continuum representation of a cellular structure analyzed here were not related to the cell size of the structure. Therefore, since the product $N V$ depends only on the ratio $k$ between the thickness and the length of a cell wall, for two different structures containing the same volume of solid material, which is distributed as a small number of large cells or as a larger number of smaller cells, respectively, such that the ratio $k$ between the thickness and the length of a wall is the same in both structures, the stiffness of the corresponding mesoscale models would be the same. While this is valid for many cellular structures with linear elastic cell walls $[16,17]$, and hence appears reasonable in the small strain regime for similar structures with nonlinear elastic cell walls as well, in many cellular solids, the cell size is expected to play a more independent role on the mechanical properties, even though this role is usually obscured by other structural characteristics, as remarked in the review article [38]. For example, in some conventional food extrudates, both the decrease of the cell size and the increase in the wall thickness were identified by Barrett et al. (1994) [1] as causing an increase in mechanical strength. Therefore, we believe that our theoretical approach should also account for the independent effect of the cell size relative to the overall size of the structure at the mesoscale level, and hence on the cells number, even though, in general, this effect is perhaps more minor than that due to the ratio between the thickness and the length of the cell wall.

To capture the independent influence of the cells number on the elastic behavior of a cellular structure under large deformations, we modify the strain-energy function (2.11) by replacing (2.10) with the following weighted mean value of the cell element 
energy:

$$
\mathcal{W}^{(c)}=\eta \mathcal{W}^{(m)}
$$

where $\eta>0$ is a positive weight chosen so that, for structures containing the same volume of solid material and having similar cell geometries, $\eta$ increases as the number of cells increases, assuming that $N V$ is fixed.

For the hyperelastic material described by (3.24), the principal components of the Cauchy stress tensor are

$$
\sigma_{i}^{(c)}=\eta \sigma_{i}^{(m)}, \quad i=1,2,3
$$

Then the nonlinear shear and elastic moduli take the form, respectively,

$$
\begin{aligned}
& \mu^{(c)}=\eta \mu^{(m)}, \\
& E^{(c)}=\eta E^{(m)} .
\end{aligned}
$$

Hence, in the small strain limit, by (3.17) and (3.7), the corresponding shear and Young's moduli are

$$
\begin{aligned}
& \mu_{0}^{(c)}=\eta(1+k)^{2} N V \mu_{0} \\
& E_{0}^{(c)}=\eta(1+k)^{2} N V E_{0},
\end{aligned}
$$

and both increase when $\eta$ increases.

In practice, for structures under tension in the third direction, the weight $\eta$ can be chosen so that the effective elastic modulus for the continuum model is equal to the effective modulus for the cell walls

$$
E_{\text {eff }}=\lim _{\alpha_{3} \rightarrow 1} \bar{E}_{\text {eff }}
$$

where $\bar{E}_{\text {eff }}$ is the slope of the effective Cauchy stress versus the effective logarithmic strain curve. We recall that the effective value of a symmetric tensor $\mathbf{s}[20,21]$ is defined as

$$
s_{\mathrm{eff}}=\sqrt{s_{11}^{2}+s_{22}^{2}+s_{33}^{2}-s_{11} s_{22}-s_{22} s_{33}-s_{33} s_{11}+3\left(s_{12}^{2}+s_{13}^{2}+s_{23}^{2}\right)} .
$$

Then

$$
\eta=\frac{E_{\text {eff }}}{(1+k)^{2} N V E_{0}} .
$$

Since cellular bodies are generally more flexible than the elastic material from which they are made, the relation $E_{0}^{(c)}<E_{0}$ is expected, in which case the condition $0<$ $\eta<1 /\left[(1+k)^{2} N V\right]$ must be satisfied. We note that, for cellular structures where the stiffness depends primarily on the ratio between the thickness and the length of the cell walls, the value of $\eta$ will be approximately equal to 1 . Nonetheless, as discussed earlier, theoretically, our continuum model seems more complete with the factor $\eta$ included than without it. 
4. Mesoscopic model for open-cells with neo-Hookean cell walls. We now specialize our model to the simple case where the cell wall material is described by the compressible neo-Hookean model

$$
\mathcal{W}\left(\lambda_{1}, \lambda_{2}, \lambda_{3}\right)=\frac{\mu}{2}\left[\lambda_{1}^{2}+\lambda_{2}^{2}+\lambda_{3}^{2}-3-2 \ln \left(\lambda_{1} \lambda_{2} \lambda_{3}\right)\right]+\frac{\lambda}{2}\left[\ln \left(\lambda_{1} \lambda_{2} \lambda_{2}\right)\right]^{2},
$$

where $\mu>0$ and $\lambda>0$ are constant parameters. The associated principal Cauchy stress components are

$$
\sigma_{i}=\frac{1}{\lambda_{1} \lambda_{2} \lambda_{3}}\left[\mu\left(\lambda_{i}^{2}-1\right)+\lambda \ln \left(\lambda_{1} \lambda_{2} \lambda_{3}\right)\right], \quad i=1,2,3 .
$$

Following the general approach described in section 2, the weighted strain-energy function at the mesoscopic scale (3.24) is

$$
\begin{aligned}
\mathcal{W}^{(c)}\left(i_{1}, i_{2}, i_{3}\right) & =\eta N V \frac{\mu}{2}\left[(1+k)^{2}\left(i_{1}^{2}-2 i_{2}\right)-2 k(1+k) i_{1}-3\left(1-k^{2}\right)\right] \\
& -\eta N V \mu \ln \left[(1+k)^{3} i_{3}-k(1+k)^{2} i_{2}+k^{2}(1+k) i_{1}-k^{3}\right] \\
& +\eta N V \frac{\lambda}{2}\left\{\ln \left[(1+k)^{3} i_{3}-k(1+k)^{2} i_{2}+k^{2}(1+k) i_{1}-k^{3}\right]\right\}^{2} .
\end{aligned}
$$

The corresponding principal Cauchy stress components are

$$
\begin{aligned}
\sigma_{i}^{(c)} & =\eta(1+k) N V \mu \frac{\alpha_{i}}{\alpha_{1} \alpha_{2} \alpha_{3}}\left[\alpha_{i}(1+k)-k-\frac{1}{\alpha_{i}(1+k)-k}\right] \\
& +\eta(1+k) N V \lambda \frac{\alpha_{i}}{\alpha_{1} \alpha_{2} \alpha_{3}} \frac{\ln \left[(1+k)^{3} i_{3}-k(1+k)^{2} i_{2}+k^{2}(1+k) i_{1}-k^{3}\right]}{\alpha_{i}(1+k)-k}, i=1,2,3 .
\end{aligned}
$$

The nonlinear shear modulus (3.26) for the cellular solid at the mesoscopic scale is

$$
\begin{aligned}
\mu^{(c)} & =\eta(1+k)^{2} N V \mu\left[1+\frac{k^{2}\left(1-2 / \sqrt{\gamma^{2}+4}\right)}{1+k(1+k)\left(2-\sqrt{\gamma^{2}+4}\right)}\right] \\
& -\eta(1+k) N V \lambda \frac{k \ln \left[(1+k)^{2}-k(1+k) \sqrt{\gamma^{2}+4}+k^{2}\right]}{\sqrt{\gamma^{2}+4}\left[(1+k)^{2}-k(1+k) \sqrt{\gamma^{2}+4}+k^{2}\right]} .
\end{aligned}
$$

In the small strain limit, the shear modulus (4.5) converges to

$$
\mu_{0}^{(c)}=\lim _{\gamma \rightarrow 0} \mu^{(c)}=\eta(1+k)^{2} N V \mu
$$

and the corresponding stretch modulus (3.27) converges to the Young's modulus

$$
E_{0}^{(c)}=\lim _{\alpha \rightarrow 1} E^{(c)}=\eta(1+k)^{2} N V E,
$$

where

$$
E=\mu \frac{2 \mu+3 \lambda}{\mu+\lambda}
$$

Then, by (3.23), the corresponding Poisson's ratio is

$$
\nu_{0}^{(c)}=\frac{\lambda}{2(\lambda+\mu)}=\nu .
$$

Copyright (c) by SIAM. Unauthorized reproduction of this article is prohibited. 
4.1. Simple compression. Next, we consider the case of simple compression of the cellular material at the mesoscopic level, such that the compression is directed parallel to one set of cell walls, as assumed also by Gent and Thomas in [15]. For circular cylinders and tubes of incompressible neo-Hookean or Mooney-Rivlin material with arbitrary length and thickness, Goriely, Vandicer, and Destrade (2008) [18] obtained an explicit formulation for the Euler elastic buckling load and its first nonlinear corrections based on the geometrical and material parameters. A similar approach was employed by DePascalis , Destrade, and Goriely (2011) [8] to derive the elastic buckling load for a compressible elastic cylinder. When a cylindrical wall of thickness $t$ and length $L=t / k$, made out of an incompressible neo-Hookean material, is subject to a simple compression, where $\lambda_{3}=\lambda<1$ and $\lambda_{1}=\lambda_{2}=1 / \sqrt{\lambda}>1$, the critical axial stretch at which buckling occurs takes the form $\lambda=1-\epsilon^{2} / 4+7 \epsilon^{4} / 36+\mathcal{O}\left(\epsilon^{6}\right)$, with $\epsilon=\pi k / 2$ small [18].

If the cell walls are similar, by (2.1)-(2.3), under the triaxial stretch diag $\left(\alpha_{1}, \alpha_{2}, \alpha_{3}\right)$ at the mesoscopic level, every cell wall deforms by a triaxial stretch $\operatorname{diag}\left(\lambda_{1}, \lambda_{2}, \lambda_{3}\right)$, where the stretches at the mesoscopic scale and at the cell wall levels are related by a rotation, depending on the cell wall orientation relative to the axial directions in which the triaxial stretch at the mesoscopic level occurs. Assuming that compression of the cellular material occurs parallel to the direction of some of the cell walls, with the orthonormal vectors $\left(\mathbf{n}_{1}, \mathbf{n}_{2}, \mathbf{n}_{3}\right)$ given by (2.2), the critical right Cauchy-Green tensor for the onset of buckling of the cell elements comprising those walls and half of a joint at each end of a wall is $\overline{\mathbf{C}}=\operatorname{diag}\left(\bar{\lambda}_{1}^{2}, \bar{\lambda}_{2}^{2}, \bar{\lambda}_{3}^{2}\right)$, where by $(2.1)$,

$$
\bar{\lambda}_{1}=\bar{\lambda}_{2}=\frac{1+k \sqrt{\lambda}}{(1+k) \sqrt{\lambda}}, \quad \bar{\lambda}_{3}=\frac{\lambda+k}{1+k} .
$$

Then, by (2.3) and (4.10), the critical principal stretches $\left\{\alpha_{i}\right\}_{i=1,2,3}$ for the cellular body at the mesoscopic scale satisfy

$$
\begin{aligned}
& \alpha_{1}=\frac{1+k \sqrt{\lambda}}{(1+k) \sqrt{\lambda}} \cos ^{2} \theta \cos ^{2} \phi+\frac{1+k \sqrt{\lambda}}{(1+k) \sqrt{\lambda}} \sin ^{2} \phi+\frac{\lambda+k}{1+k} \sin ^{2} \theta \cos ^{2} \phi, \\
& \alpha_{2}=\frac{1+k \sqrt{\lambda}}{(1+k) \sqrt{\lambda}} \cos ^{2} \theta \sin ^{2} \phi+\frac{1+k \sqrt{\lambda}}{(1+k) \sqrt{\lambda}} \cos ^{2} \phi+\frac{\lambda+k}{1+k} \sin ^{2} \theta \sin ^{2} \phi, \\
& \alpha_{3}=\frac{1+k \sqrt{\lambda}}{(1+k) \sqrt{\lambda}} \sin ^{2} \theta+\frac{\lambda+k}{1+k} \cos ^{2} \theta .
\end{aligned}
$$

For the mesoscopic model, the corresponding critical Cauchy stress is then computed from (2.13) and (3.25). In particular, in the Euler buckling limit $\lambda \rightarrow 1$, the critical compressive stress predicted by the linear-elastic models [15] is obtained.

5. Numerical examples. In section 2 , the unit volume for the continuum model was considered as a sphere centered at the intersection of adjacent walls. However, the results are valid also if the unit volume is cubical in shape with the cuboid walls aligning with the cube edges and the cubical joints placed at the cube corners. Moreover, the generality of the theoretical model, which is not restricted to a specific cell wall orientation, enables us to derive mesoscopic models corresponding to both three- and two-dimensional structures, with different cell geometries, by applying the same approach. In this section, we test the qualitative performance of the mesoscopic hyperelastic models in the case of computer simulations of three-dimensional periodic open-cell structures with cubical cells and of two-dimensional honeycombs with hexagonal cells of neo-Hookean material under large tension. 
The structural models presented here were created in SolidWorks and imported into the Finite Elements for Biomechanics (FEBio) software [24], and a mesh refinement study was performed for each structure, so that the numerical results are independent of the mesh-size. Each structure is formed from a single piece of solid material described by the compressible neo-Hookean strain-energy function (4.1), with $\mu=E /[2(1+\nu)]$ and $\lambda=\nu E /[(1+\nu)(1-2 \nu)]$, where $E=0.1 \mathrm{MPa}$ and $\nu=0.49$, and is subject to finite tension with the following boundary conditions: the lower external horizontal face is fixed in the $Y$ /second/vertical-direction and free to slide in the $X /$ first/horizontal-direction and in the $Z /$ third/out-of-plane-direction; the upper external horizontal face is under prescribed tension in the $Y$-direction and is free to slide in the $X$ and $Z$-directions; the remaining external and internal cell faces deform freely.

For these structures, the mean effective Cauchy stress (normalized by $E=0.1$ $\mathrm{MPa}$ ) versus the mean effective logarithmic strain in the cell walls, and also versus the prescribed mesoscale tensile deformation, is shown together with the effective Cauchy stress for the associated continuum models. The mean value was calculated as the sum of the values on all the finite elements divided by the number of elements. For the numerical examples involving three-dimensional periodic structures with cubical cells and two-dimensional honeycombs presented here, we conclude that if the deformation of the cell walls is close to the triaxial stretch and the joints do not deform significantly, as assumed theoretically, then the continuum models offer a good approximation for the respective structures.

5.1. Open-cell structures with cubical cells. We consider six open-cell structures with cubical cells in stacked distribution, as shown in Figures 2 and 4, with the corresponding geometric parameters specified in Tables 1 and 2, respectively. These structures are equal in size in the undeformed state, i.e., they occupy equal cubical volumes, and are subject to equal prescribed tensile deformations, up to $20 \%$, in the vertical direction.

In each structure, six cell walls, with undeformed thickness $t$ and length $L$, are meeting at a common joint of surface area $6 t^{2}=6 k^{2} L^{2}$, where $k=t / L$. Taking a representative volume of the cellular material scale in the undeformed state as a cube of side $L+t=L(1+k)$, the volume of this cube is $\bar{V}_{m}=(L+t)^{3}=L^{3}(1+k)^{3}$, while the volume of solid material contained in this cube is $\bar{V}=6 t^{2}(L / 2)+t^{3}=k^{2} L^{3}(3+k)$. Hence the volume ratio is

$$
\bar{V} / \bar{V}_{m}=1-\frac{1+3 k}{(1+k)^{3}},
$$

and is equal to that given by (2.16). Setting $\bar{V}_{m}=1$, the volume fraction of cell wall material in this cube is then equal to that given by (2.17), i.e.,

$$
N V=\frac{6 t^{2} L}{2}=\frac{3 k^{2}}{(1+k)^{3}} \text {. }
$$

5.1.1. Fixed number of cells and increasing wall thickness. First, we consider the three structures with a fixed number of cells and increasing wall thickness illustrated in Figure 3, for which the corresponding geometric parameters are recorded in Table 1. For these structures, the numerical results show that the Cauchy stress increases as the deformation increases, and both the mean effective Cauchy stress and the mean effective strain are greater in the structures with thicker cell walls than in those with thinner walls (see Figure 3 (a)). Furthermore, at equal mesoscale deformations, the slope of the curve representing the mean effective Cauchy stress versus 

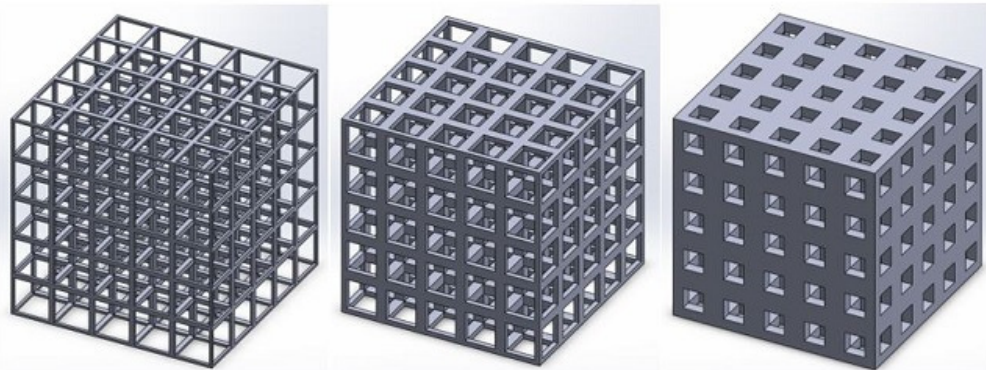

FIG. 2. Undeformed periodic open-cell cubes with thin (left), medium (middle), and thick (right) cell walls and a fixed number of cells.
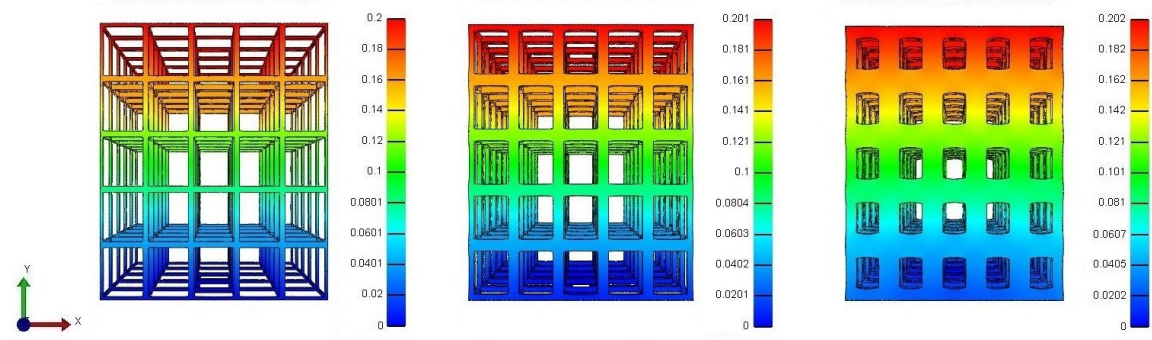

(a)

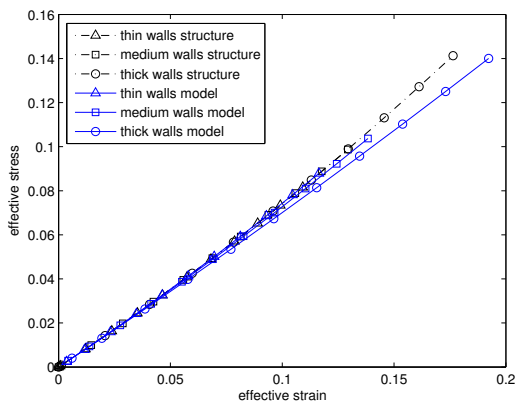

(b)

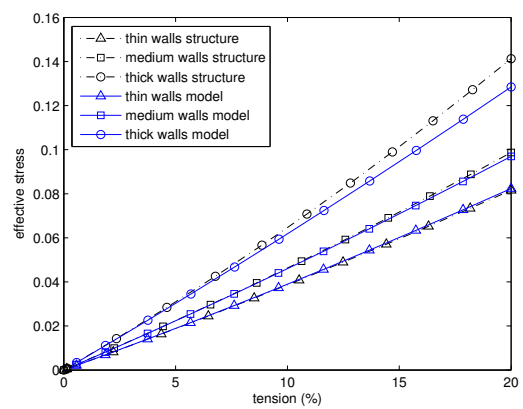

FIG. 3. Comparisons between the effective Cauchy stress for the mesoscale model and the cubical structures with a fixed number of cells and different cell wall thickness versus (a) the effective strain and (b) the mesoscale deformation. Above, the cubical structures with thin (left), medium (middle), and thick (right) cell walls are illustrated at $20 \%$ tension in the vertical direction (color bar showing the displacement in the same direction).

the mesoscopic tensile deformation increases as the thickness of the cell wall increases (see Figure 3 (b)). The continuum models approximating these structures also capture these behaviors, and under large strains, appear more accurate for the structures with thinner walls, where the deformation is closer to the triaxial stretch and the joints deform less significantly, as assumed theoretically, than for the structures with thicker walls.

5.1.2. Constant volume and increasing number of cells. Next, we look at the effect of increasing the number of cells under the constraint of keeping both the total volume of solid material and the cell wall thickness to length ratio constant, as shown in Figure 5, with the respective geometric parameters given in Table 2. In this case, for the three different structures, the mean effective Cauchy stress increases as 
TABLE 1

Geometric parameters for the undeformed periodic open-cell structures with a fixed number of cubical cells and different wall thickness. In the undeformed state, each structure occupies a cube domain of dimensionless side 1 (see Figure 2).

\begin{tabular}{c|c|c|c|c|c}
\hline Structure & Number of cells & Volume of solid material & Wall length $L$ & Wall thickness $t$ & $k=t / L$ \\
\hline thin walls & 125 & 0.0781 & 0.1750 & 0.0250 & 0.1429 \\
medium walls & 125 & 0.1562 & 0.1500 & 0.0500 & 0.3333 \\
thick walls & 125 & 0.3124 & 0.1000 & 0.1000 & 1.0000 \\
\hline
\end{tabular}

TABLE 2

Geometric parameters for the undeformed periodic open-cell structures with fixed material volume and an increasing number of cubical cells. In the undeformed state, each structure occupies a cube domain of dimensionless side 1 (see Figure 4).

\begin{tabular}{c|c|c|c|c|c}
\hline Structure & Number of cells & Volume of solid material & Wall length $L$ & Wall thickness $t$ & $k=t / L$ \\
\hline $3 \times 3 \times 3$ cells & 27 & 0.1562 & 0.2500 & 0.0833 & 0.3333 \\
$5 \times 5 \times 5$ cells & 125 & 0.1562 & 0.1500 & 0.0500 & 0.3333 \\
$9 \times 9 \times 9$ cells & 729 & 0.1562 & 0.0833 & 0.0278 & 0.3333 \\
\hline
\end{tabular}

the deformation increases, and the slope of the curves representing the mean effective Cauchy stress versus the mean effective strain in the cell walls are equal (see Figure 5 (a)), which is in agreement with the results for stretch-dominated structures from the linear elasticity theory [17]. The numerical results also show that both the mean effective Cauchy stress and the mean effective strain are greater in the structures with a larger number of small cells than in the ones with a smaller number of large cells, but they provide little insight into the performance of the continuum models in approximating the behavior of individual structures under large strains. In addition, the slope of the curve representing the mean effective Cauchy stress versus the mesoscale tensile deformation, increases as the number of cells increases, even though both the volume of solid material and the ratio between the thickness and the length of the cells are the same for the three different structures (see Figure $5(\mathrm{~b})$ ). The accuracy of the continuum models capturing these behaviors appears similar for the three structures.

5.2. Honeycomb structures. We further assess the numerical performance of the continuum models developed here in approximating quasi-planar honeycomb structures with hexagonal cells. For the three structures shown in the undeformed state in Figure 6, the geometric parameters are listed in Table 3. These honeycomb structures are taken equal in size in the undeformed state, i.e., they occupy equal overall volumes, and are deformed by up to $20 \%$ tension in the vertical direction. However, for the three different structures, with an increasing number of cells, the overall volumes of solid material and the ratios between the thickness and the length of the walls are different in the undeformed state.

TABLE 3

Geometric parameters for the undeformed periodic honeycomb structures. In the undeformed state, each structure occupies a square domain of dimensionless side 1 (see Figure 6).

\begin{tabular}{c|c|c|c|c}
\hline Structure & Number of cells & Wall length $L$ & Wall thickness $t$ & $k=t / L$ \\
\hline large cells & 8 & 0.1570 & 0.0613 & 0.3904 \\
medium cells & 23 & 0.0866 & 0.0500 & 0.5774 \\
small cells & 77 & 0.0495 & 0.0254 & 0.5131 \\
\hline
\end{tabular}

In these structures, three cell walls of undeformed thickness $t$ and length $L$ are meeting at a common joint of surface area $3 t^{2}=3 k^{2} L^{2}$. Hence the cell wall material 

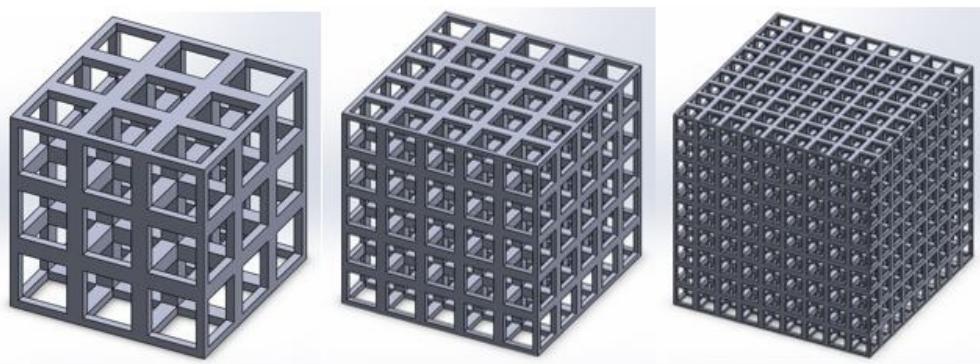

FIG. 4. Undeformed periodic open-cell cubes with $3 \times 3 \times 3$ (left), $5 \times 5 \times 5$ (middle), and $9 \times 9 \times 9$ (right) cells and fixed material volume.
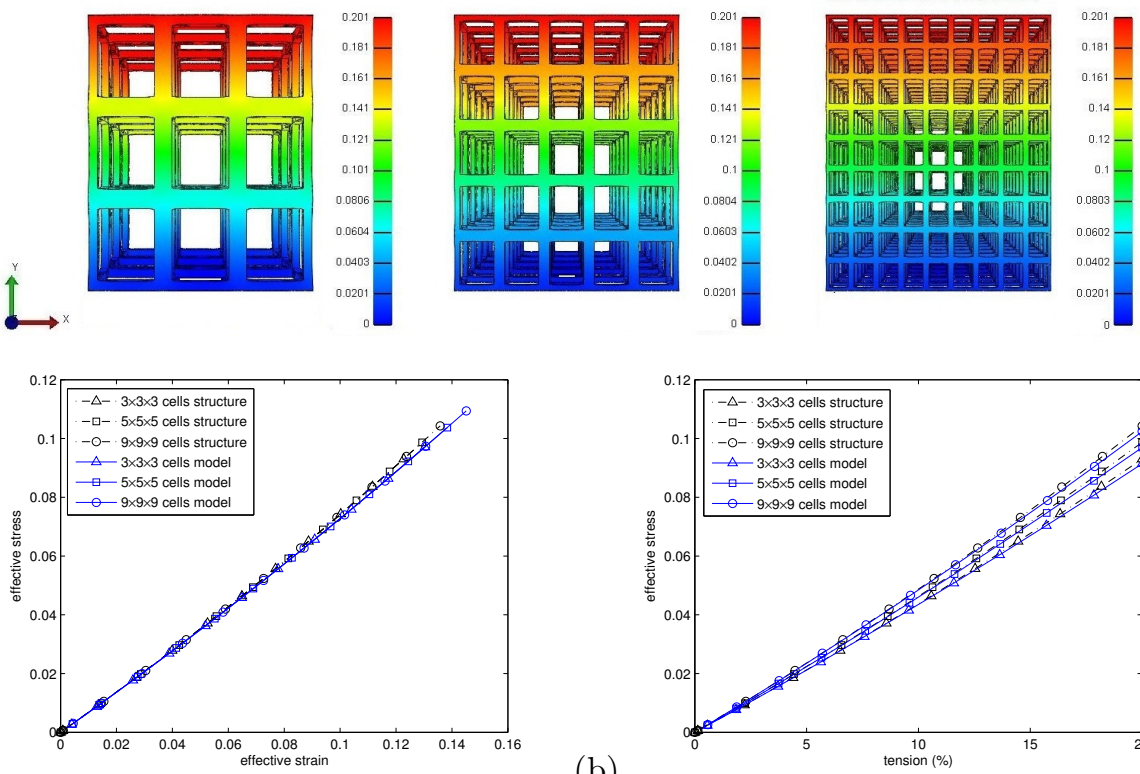

(a)

(b)

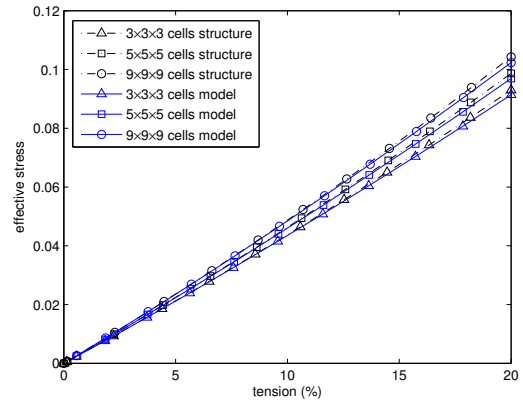

FIG. 5. Comparisons between the effective Cauchy stress for the mesoscale model and the cubical structures with fixed material volume and an increasing number of cells versus (a) the effective strain and (b) the mesoscale deformation. Above, the cubical structures with $3 \times 3 \times 3$ (left), $5 \times 5 \times 5$ (middle), and $9 \times 9 \times 9$ (right) cells are shown at $20 \%$ tension in the vertical direction (color bar representing the displacement in the same direction).

contained in a unit cube of side $L+t=L(1+k)$ is

$$
N V=\frac{3 t^{2} L}{2}=\frac{3 k^{2}}{2(1+k)^{3}},
$$

and differs from (2.17) by a factor of 2. In Figure 7, the Cauchy stress for the continuum models shows good agreement with the Cauchy stress in the deforming structures. In this case also, the stress increases as the deformation increases, and at a fixed mesoscale deformation, is greater in the structures with a larger number of small cells than the ones with a smaller number of large cells.

6. Conclusion. Homogeneous isotropic hyperelastic models for stretch-dominated open-cell structures, at a mesoscopic level, where the number of cells is finite and the 

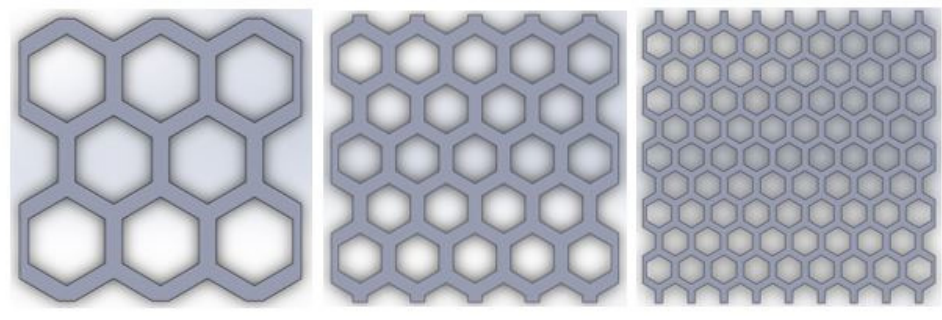

FIG. 6. Undeformed periodic honeycomb structures with large (left), medium (middle), and small (right) hexagonal cells.
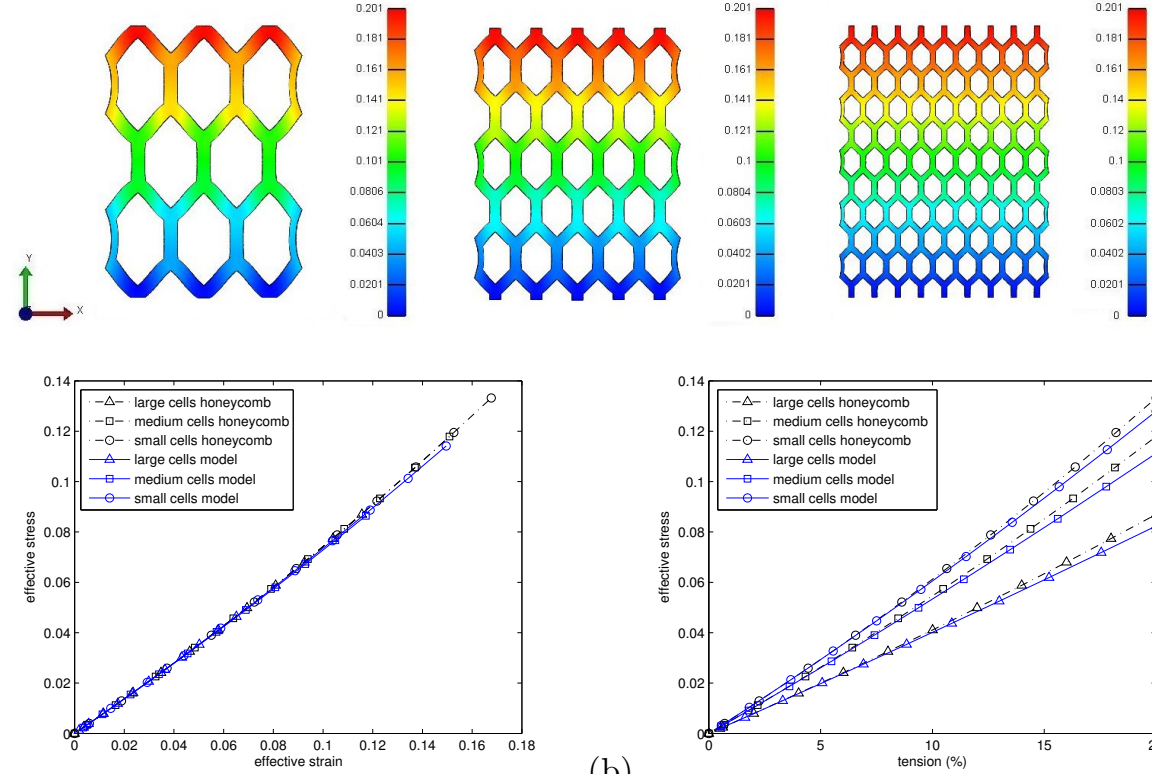

(b)

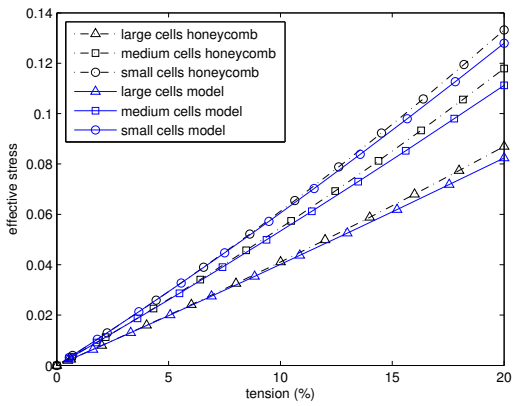

FIG. 7. Comparisons between the effective Cauchy stress for the mesoscale model and for the honeycomb structures versus (a) the effective strain and (b) the mesoscale deformation. Above, the honeycomb structures with large (left), medium (middle), and small (right) cells are represented at $20 \%$ tension in the vertical direction (color bar showing the displacement in the same direction).

size of the structure is comparable to the size of the cells, were derived from the architecture and the material constitutive law of the constituents, and their material parameters were predicted from the strain-energy function and the large strains in the cell walls.

Theoretically, the continuum models developed here are applicable to irregular structures, with arbitrarily oriented cell walls made from a general isotropic nonlinear hyperelastic material. Numerical results show that the hyperelastic models capture well the mechanical responses of finite element simulations for three-dimensional periodic structures and for two-dimensional honeycombs with a finite number of cells made from a neo-Hookean material under large tension. Moreover, due to the generality of the theoretical model, which is not restricted to a specific cell wall orientation, the mesoscopic models corresponding to three- and two-dimensional structures with different cell geometries can be derived by applying the same approach.

The proposed models for open-cell structures can be enhanced to account for the 
behavior of cellular structures with closed cells, and further extended to composite cellular structures where the cells are filled with a hyperelastic core [32]. In computer simulations where modelling a very large number of cells individually is not feasible, continuum models can be incorporated in multiscale approaches which significantly reduce computational cost and may even mean the difference between finding a solution or not.

Acknowledgments. The authors would like to thank Professor Robin Knops of Heriot-Watt University, Edinburgh, for recommending reference [4]. The support provided to L. Angela Mihai and Hayley Wyatt by the Engineering and Physical Sciences Research Council of Great Britain under research grant EP/M011992/1 is gratefully acknowledged.

\section{REFERENCES}

[1] A. H. Barett, A. V. Cardello, L. L. Lesher, and I. A. Taub (1994), Cellularity, mechanical failure, and textural perception of corn meal extrudates, J. Texture Studies, 25, pp. 77-95, https://doi.org/10.1111/j.1745-4603.1994.tb00756.x.

[2] M. F. BEATTY (1989), Gent-Thomas and Blatz-Ko models for foamed elastomers, in Mechanics of Cellulosic and Polymeric Materials, AMD Vol. 99 (MD Vol. 13), R. W. Perkins, ed., American Society of Mechanical Engineering, New York, pp. 75-78.

[3] M. F. BEATty (1996), Introduction to nonlinear elasticity, in Nonlinear Effects in Fluids and Solids, M. M. Carroll and M. A. Hayes, eds., Plenum, New York, pp. 13-104.

[4] M. F. Beatty (2001), Seven lectures in finite elasticity, in Topics in Finite Elasticity, M. Hayes and G. Saccomandi, eds., Springer-Verlag, Wien, pp. 31-93.

[5] M. F. Beatty and D. O. Stalnaker (1986), The Poisson function of finite elasticity, J. Appl. Math., 53, 99. 807-813, https://doi.org/10.1115/1.3171862.

[6] A. Bhaskar (2009), The effective Poisson ratio of random cellular matter having bending dominated architecture, Europhys. Lett., 87, 18004, https://doi.org/10.1209/0295-5075/ $87 / 18004$.

[7] P. J. Blatz and W. L. Ko (1962), Application of finite elastic theory to deformation of rubbery materials, Trans. Soc. Rheology, 6, pp. 223-251.

[8] R. De Pascalis, M. Destrade, and A. Goriely (2011), Nonlinear correction to the Euler buckling formula for compressed cylinders with guided-guided end conditions, J. Elasticity, 102, pp. 191-200, https://doi.org/10.1007/s10659-010-9265-6.

[9] V. S. Deshpande, M. F. Ashby, And N. A. Fleck (2001), Foam topology: bending versus stretching dominated architectures, Internat. J. Solids Structures, 49, pp. 1035-1040, https: //doi.org/10.1.1.576.9756.

[10] V. S. Deshrande, N. A. Fleck, and M. F. Ashby (2001), Effective properties of the octettruss lattice material, J. Mech. Phys. Solids, 49, pp. 1747-1769, https://doi.org/10.1016/ S0022-5096(01)00010-2.

[11] G. C. Engelmayr, Jr., G. D. Papworth, S. C. Watkins, J. E. Mayer, Jr., and M. S. SACKS (2006), Guidance of engineered tissue collagen orientation by large-scale scaffold microstructures, J. Biomech., 39, pp. 1819-1831, https://doi.org/10.1016/j.jbiomech.2005. 05.020.

[12] M. A. Fortes And M. T. Nogueira (1989), The Poisson effect in cork, Mater. Sci. Eng. A, 122, pp. 227-232, https://doi.org/10.1016/0921-5093(89)90634-5.

[13] M. Fournier, J. Dlouhá, G. Jaouen, and T. Almeras (2013), Integrative biomechanics for tree ecology: beyond wood density and strength, J. Exp. Bot., 64, pp. 4793-4815, https: //doi.org/10.1093/jxb/ert279.

[14] A. N. Gent and A. G. Thomas (1959), The deformation of foamed elastic materials, J. Appl. Polymer Science, 1, pp. 107-113, https://doi.org/10.1002/app.1959.070010117.

[15] A. N. Gent and A. G. Thomas (1963), Mechanics of foamed elastic materials, Rubber Chem. Tech., 36, pp. 597-610, https://doi.org/10.5254/1.3539591.

[16] L. J. Gibson And M. F. Ashby (1997), Cellular Solids: Structure and Properties, 2nd ed, Cambridge University Press, Cambridge, UK, https://doi.org/10.1017/CBO9781139878326.

[17] L. J. Gibson, M. F. Ashby, And B. A. Harley (2010), Cellular Materials in Nature and Medicine, Cambridge University Press, Cambridge, UK. 
[18] A. Goriely, R. Vandiver, and M. Destrade (2008), Nonlinear Euler buckling, Proc. R. Soc. Lond. Ser. A Math. Phys. Eng. Sci., 464, pp. 3003-3019, https://doi.org/10.1098/rspa. 2008.0184.

[19] R. Hill (1978), Aspects of invariance in solid mechanics, Advances in Applied Mechanics 18, Academic Press, New York, London, pp. 1-75, https://doi.org/10.1016/S0065-2156(08) 70264-3.

[20] J. W. Hutchinson and K. W. Neale (1981), Finite strain $J_{2}$ deformation theory, Proceedings of the IUTAM Symposium on Finite Elasticity, Martinus Nijhoff, Netherlands.

[21] D. Jung And H. C. Gea (2004), Topology optimization of nonlinear structures, Finite Elem. Anal. Des., 40, pp. 1417-1427, https://doi.org/10.1016/j.finel.2003.08.011.

[22] A. E. H. Love (1927), A Treatise on the Mathematical Theory of Elasticity, Cambridge University Press, Cambridge, UK.

[23] P. Le TAllec (1994), Numerical methods for three-dimensional elasticity, in Handbook of Numerical Analysis, Vol. III, P. G. Ciarlet and J. L. Lions, eds., North-Holland, Amsterdam, pp. $465-622$.

[24] S. A. Maas, B. J. Ellis, G. A. Ateshian, and J. Weiss (2012), FEBio: Finite Elements for Biomechanics, J. Biomech. Eng., 134, 011005, https://doi.org/10.1115/1.4005694.

[25] L. A. Mihai, K. Alayyash, and A. Goriely (2015), Paws, pads, and plants: The enhanced elasticity of cell-filled load-bearing structures, Proc. A, 471, 20150107, https://doi.org/10. 1098/rspa.2015.0107.

[26] L. A. Mihai, K. Alayyash, and H. Wyatt (2016), The optimal density of cellular solids in axial tension, Comput Methods Biomech Biomed Engin., 20, pp. 701-713, https://doi.org/ 10.1080/10255842.2017.1292352.

[27] L. A. Mihai and A. Goriely (2014), Nonlinear Poisson effects in soft honeycombs, Proc. R. Soc. A, 470, 20140363, https://doi.org/10.1098/rspa.2014.0363.

[28] L. A. Minai and A. Goriely (2015), Finite deformation effects in cellular structures with hyperelastic cell walls, Internat. J. Solids and Structures, 53, pp. 107-128, https://doi.org/ 10.1016/j.ijsolstr.2014.10.015.

[29] L. A. Minai and P. NefF (2017), Hyperelastic bodies under homogeneous Cauchy stress induced by non-homogeneous finite deformations, Internat. J. Non-Linear Mech., 89, pp. 93100, https://doi.org/10.1016/j.ijnonlinmec.2016.12.003.

[30] L. A. Minai and P. NeFF (2017), Hyperelastic bodies under homogeneous Cauchy stress induced by three-dimensional non-homogeneous deformations, Math. Mech. Solids, https: //doi.org/10.1177/1081286516682556.

[31] L. A. Mihai, A. Safar, And H. Wyatt (2017), Debonding of cellular structures with fibrereinforced cell walls under shear deformation, J. Eng. Math., pp. 1-17, https://doi.org/ 10.1007/s10665-016-9894-2.

[32] L. A. Mihai, H. Wyatt, and A. Goriely (2017), Microstructure-based hyperelastic models for closed-cell solids, Proc. Math. Phys. Eng. Sci., 473, 20170036, https://doi.org/10.1098/ rspa.2017.0036.

[33] P. NefF And L. A. Minai (2016), Injectivity of the Cauchy-stress tensor along rank-one connected lines under strict rank-one convexity condition, J. Elasticity, 127, pp. 309-315, https://doi.org/10.1007/s10659-016-9609-y.

[34] J. T. Oden (2006), Finite Elements of Nonlinear Continua, 2nd ed, Dover, Mineola, NY.

[35] R. W. OGden (1972), Large deformations isotropic elasticity - on the correlation of theory and experiment for incompressible rubberlike solids, Proc. R. Soc. A, 326, pp. 565-584, https://doi.org/10.1098/rspa.1972.0026.

[36] R. W. Ogden (1997), Non-Linear Elastic Deformations, 2nd ed, Dover, Mineola, NY.

[37] P. M. Rich (1986), Mechanical architecture of arborescent rain forest palms, Principes, 30, pp. $117-131$.

[38] M. G. Scanlon (2005), Biogenic cellular solids, in Soft Materials: Structure and Dynamics, J. R. Dutcher and A. G. Marangoni, eds., Marcel Dekker, New York, pp. 321-349.

[39] A. J. M. Spencer (1973), Theory of invariants, in Continuum Physics, Vol. 1, A. C. Eringen, ed., Academic Press, New York, pp. 239-353.

[40] B. Storakers (1986), On the material representation and constitutive branching in finite compressible elasticity, J. Mech. Phys. Solids, 34, pp. 125-145, https://doi.org/10.1016/ 0022-5096(86)90033-5.

[41] C. Truesdell and W. Noll (2004), The Non-Linear Field Theories of Mechanics, 3rd ed, Springer-Verlag, Berlin.

[42] D. Weaire And M. A. Fortes (1994), Stress and strain in liquid and solid foams, Adv. Phys., 43, pp. 685-738, https://doi.org/10.1080/00018739400101549.

Copyright (c) by SIAM. Unauthorized reproduction of this article is prohibited. 